\title{
Validación de un instrumento de ansiedad dental EQ-SDAI
}

\author{
Validation of an instrument of dental anxiety EQ-SDAI \\ Ebingen Villavicencio ${ }^{\text {1a }}$, Dayana Castillo ${ }^{\text {1b }}$, Maribel Llapa ${ }^{\text {1b }}$, Zoila Jaramillo ${ }^{\text {1b }}$, Paúl Coronel ${ }^{\text {bb }}$, \\ María del Carmen Pariona ${ }^{1 c}$.
}

\section{RESUMEN}

Objetivo: El objetivo de este estudio fue validar un instrumento de uso masivo para evaluar la ansiedad dental, mediante la dicotomización de una variable continua del Instrumento de versión corta del Inventario de Ansiedad Dental (SDAI) a una variable binaria del instrumento EQ-SDAI. Material y métodos: Se utilizó una población de edad > 18 años en el análisis. El SDAI se adaptó interculturalmente al español y se llamó EQ-SDAI; luego se calculó la sensibilidad y la especificidad. También se utilizó la curva ROC para evaluar la confiabilidad del nuevo cuestionario. Se calculó el coeficiente de correlación biserial puntual. Para el análisis de consistencia interna se utilizó el coeficiente Kuder Richardson (KR-20). Resultados: Se obtuvo una alta sensibilidad (Sen $=0,93)$ y una especificidad aceptable $(E s p=0,60)$. A través del punto de coeficiente de correlación biserial, se obtuvo una buena correlación ( $\mathrm{R}>0,70)$, en ocho de las nueve preguntas de la escala SDAI. Conclusiones: Los resultados indican que existe un buen coeficiente de correlación. La consistencia interna (KR-20) fue 0,866.

PALABRAS CLAVE: Ansiedad dental, estudios de validación, salud bucal, atención dental, encuestas y cuestionarios.

\section{SUMMARY}

Objective: The aim of this study was to validate an instrument of mass use to evaluate dental anxiety, by means of the dichotomization of a continuous variable of the Dental Anxiety Inventory short version instrument (SDAI) to a binary variable of the EQ- SDAI instrument. Material and Methods: One population aged $>18$ years were used in the analysis. The SDAI was cross-culturally adapted to Spanish, and was named EQ-SDAI, then sensibility and specificity was calculated also the ROC curve was used to assess the reliability of the new questionnaire. Point biserial correlation coefficient was calculated. For the internal consistency analysis, the Kuder Richardson coefficient (KR-20) was used. Results: High sensitivity $(\mathrm{Sen}=0.93)$, and an acceptable specificity $(\mathrm{Esp}=0.60)$ was obtained. Through the point biserial correlation coefficient, a statistically significant result $(\mathrm{R}>0.70)$ was obtained, in eight of the nine questions of the SDAI scale, which indicates that there is a good coefficient of correlation. Conclusions: The internal consistency (KR-20) was 0.866.

KEY WORDS: Dental anxiety, validation studies, oral health, dental care, surveys and questionnaires.

Facultad de Odontología, Universidad Católica de Cuenca. Cuenca, Ecuador.

Coordinador de investigación; PhD.

Odontólogo de consulta particular; DDS.

Profesora de Odontopediatría; DDS, Especialista. 


\section{INTRODUCCIÓN}

En investigación científica aplicada al campo de la salud bucal, las encuestas representan un componente importante para la recolección de datos, que permiten determinar la condición de salud bucodental presente y posibles necesidades a futuro (1). La validación de un instrumento depende de su capacidad para medir la variable que se desea medir (validez) $(2,3,4)$, mientras que su idoneidad para que pueda repetir el valor de la medición, en un mismo individuo y bajo las mismas circunstancias, pero en un periodo de tiempo diferente, es la fiabilidad del instrumento (llamada también estabilidad) $(2,4,5,6)$. Verificar la validez de un instrumento en la fase de prueba piloto es también esencial para identificar y corregir posibles sesgos (7).

Para el análisis de datos de un cuestionario en el campo de la salud, se emplean estadísticos que ayuden a evaluar el nivel de la enfermedad en cuestión y se realiza la dicotomización de una variable continua (8), convirtiéndola en una variable dicotómica cuando se pretende estimar la frecuencia o prevalencia de un fenómeno $(9,10)$. En la literatura de epidemiología y estadística, se prefiere dicotomizar las variables en una versión más simple, para reportar riesgo, exposición o la presencia de enfermedad ("alto" y "bajo"; "presencia" y "ausencia"), gracias a que este tipo de dato ayuda a la mejor aplicación de los diseños epidemiológicos y la estimación del riesgo $(11,12)$.

Las pruebas estadísticas recomendadas para la dicotomización de las variables continuas son, la correlación biserial (9) (entre el patrón de respuestas obtenidas por cada ítem y la puntuación total resultante de la suma de las respuestas a todos los ítems); la sensibilidad / especificidad y el cálculo del estadístico Alfa de Kuder Richardson (KR-20) (13).

La ansiedad al tratamiento dental es también conocida como odontofobia $(14,15)$, es un fenómeno multifactorial definido como el miedo subjetivo, exagerado y permanente ante un evento anticipado o presente que involucra al odontólogo, instrumental y equipo dental o al tratamiento bucodental respectivo (16).

La ansiedad al tratamiento dental con frecuencia da paso al deterioro de la salud bucal (14), actúa como una barrera para el acceso a servicios de salud estomatológica (17). Por lo tanto, una valoración acertada de la ansiedad dental, es imprescindible para disminuir las posibilidades de que el paciente deje de acudir de forma regular a las consultas con el odontólogo o de que en el peor de los casos abandone el tratamiento (18). A estos instrumentos se les conoce como pruebas de diagnóstico clínico (19).

Actualmente se necesita un instrumento epidemiológico de evaluación poblacional para la ansiedad al tratamiento dental, rápido y corto, que permita identificar de forma masiva y económica a las personas con esta condición, para poder analizar el porcentaje de la población que presenta tal padecimiento, y en el caso de existir una alta frecuencia de este fenómeno, se evidencie la necesidad de capacitar a los odontólogos para que puedan hacer frente a este reto, dado que únicamente existen instrumentos clínicos aplicables a un pequeño grupo poblacional, que ya están en plena atención dental, olvidando la posibilidad de identificar a las personas que no acceden al servicio por este motivo. La principal relevancia de este estudio se evidencia por la utilidad de dotar a la comunidad científica odontológica de un instrumento modificado y ajustado por la necesidad de tener una encuesta epidemiológica.

Este estudio tuvo el propósito de colaborar con el sistema de salud, poniendo a disposición un instrumento que permita evidenciar la magnitud de la frecuencia de personas con ansiedad dental, y que probablemente no asisten a consulta por este motivo. El objetivo del presente estudio fue validar un instrumento de uso masivo para evaluar la ansiedad dental, por medio de la dicotomización de una variable continua del instrumento Escala de Ansiedad Dental versión corta (SDAI) a una variable binaria del instrumento EQ-SDAI.

\section{MATERIAL Y MÉTODOS}

Se llevó a cabo un estudio transversal que recogió información a través de encuestas, entre pacientes de edades desde 18 a 65 años, donde se comparan los resultados obtenidos en la escala SDAI de respuestas politómicas con escala de Likert, frente a la EQSDAI con respuestas dicotómicas. El tamaño de la muestra tanto en el SDAI como en el EQ-SDAI fue 
de 696. Los pacientes fueron seleccionados de manera aleatoria entre las personas encuestadas en la prueba piloto del II Macro estudio epidemiológico llevado a cabo por la carrera de Odontología de la Universidad Católica de Cuenca, en 15 parroquias urbanas de la ciudad en los años 2017 - 2018.

Los criterios de selección utilizados para este estudio fueron: personas entre 18 a 65 años, que no presenten discapacidad motora, que no sean médicos ni odontólogos, que acepten voluntariamente participar en el estudio firmando el consentimiento informado, que tengan suficiente capacidad para leer la encuesta y responderla.

La versión (original) SDAI con respuestas politómicas contiene 5 respuestas de diferentes niveles (siempre, muy frecuente, algunas veces, pocas veces y nunca), mientras que la versión con respuestas dicotómicas (EQ-SDAI) contiene únicamente 2 respuestas (SI y NO).

Se analizaron los resultados de la versión de respuestas dicotómicas (EQ-SDAI), por medio del coeficiente de correlación biserial puntual $(20,21,22)$, en la cual se asignaron valores cuantitativos de 0 para $\mathrm{NO}$ y de 1 para SI, en cada una de las preguntas, con lo cual la escala total podría ir desde 0 hasta 9; al realizar esta suma de todas las preguntas del EQ-SDAI se categorizó a los pacientes con valor 0 como paciente sin ansiedad y todos los demás como pacientes con ansiedad, valores iguales o mayores a 1 indican que se trata de un paciente con ansiedad al tratamiento dental. Como segunda fase de validación, se empleó la estrategia de determinar la similitud de diagnóstico de sanos/enfermos de la encuesta EQ-SDAI comparada con la encuesta SDAI (Gold Estándar) para verificar la validez de la dicotomización del instrumento propuesto, esto se realizó mediante el análisis de la sensibilidad y especificidad $(23,24,25)$. A manera de comprobación de las proporciones obtenidas con uno y otro método, se empleó el estadístico $\mathrm{X}^{2}$ para comparar prevalencias (26).

Posteriormente se usó el método de Curvas ROC (Receiver Operating Characteristic Curve) (gráfico 1) (27), para delimitar el punto de corte que tenga la sensibilidad y especificidad más elevada y además, para determinar la capacidad que tiene el cuestionario de diagnóstico propuesto para discriminar a las personas sin enfermedad de las que están enfermas $(28,29,30,31)$, en donde en el eje $\mathrm{X}$ se reporta 1especificidad y en el eje Y la sensibilidad, lo que permite evaluar que cuando la sensibilidad aumenta también aumenta la especificidad (27).

En base a la escala SDAI de Stouthard, Groen y Mellenbergh, construida en $1995(14,16)$ se dicotomizó la escala teniendo como punto de corte el dato menor igual a 13 puntos, que el autor considera como pacientes sin ansiedad y valores superiores como pacientes con ansiedad (16). Paralelamente los valores de la escala de EQ-SDAI también fueron dicotomizados, como se describió anteriormente. Finalmente para el análisis de consistencia interna se utilizó el coeficiente Kuder Richardson (KR20), que es una particularidad matemática del Alfa de Cronbach utilizada para medir la consistencia interna de una escala cuando las variables son dicotómicas $(32,33,34)$. Todos los pacientes dieron su consentimiento informado para realizar este estudio, los investigadores, garantizaron la confidencialidad de los datos. La autora de la validación original del SDAI (14), dio su autorización, por escrito para el uso de esta escala.

\section{RESULTADOS}

La muestra de este estudio estuvo conformada por 371 mujeres y 325 hombres.

Para la validación del cuestionario EQ-SDAI, se emplearon varios estadísticos (Chi Cuadrado, KR-20, ANOVA, HSD-Tukey) con el propósito de comparar los resultados del EQ-SDAI con el cuestionario SDAI versión corta aplicado a la misma muestra. Para verificar la sensibilidad y especificidad del EQSDAI se utilizó el Alfa de Kuder Richardson (KR20). El valor de KR-20 hallado en el presente estudio se considera con un nivel bueno $\left(\mathrm{KR}_{20}=0,866\right)$.

La prevalencia de ansiedad dental respecto al sexo femenino y valorada a través de la escala EQ-SDAI fue de $83 \%$, en tanto que la evaluada con el SDAI fue del 85\%, al comparar estas prevalencias con la prueba estadística Chi Cuadrado, la diferencia no fue estadísticamente significativa $(\mathrm{p}=0,48)$ (tabla 1). Respecto al sexo masculino la prevalencia de acuerdo a la escala EQ-SDAI fue de $75 \%$ y con la escala SDAI fue del $79 \%$, al comparar estas prevalencias no 
se encontró diferencia estadísticamente significativa mediante $\mathrm{X}^{2}(\mathrm{p}=0,27)($ tabla 1$)$.

La prevalencia de Ansiedad Dental con la escala EQSDAI fue del $79 \%$, en tanto que la prevalencia con el SDAI fue de $82 \%$; lo cual no mostró diferencia estadísticamente significativa mediante $X^{2}(p=0,19)$ (tabla 2).

Al comparar los promedios de los puntajes del EQSDAI en los distintos niveles que plantea el SDAI, mediante la prueba estadística ANOVA, se encontró que los grupos no son iguales, lo cual luego condujo a evaluar si los grupos de ambos instrumentos son equivalentes, para lo cual posteriormente se realizó la prueba HSD-Tukey, con la finalidad de saber si existe entrecruzamiento entre los grupos y se determinó que no hay entrecruzamiento, ya que cada uno de los grupos actúa como si fuera un subgrupo distinto y la diferencia es estadísticamente significativa entre los grupos (tabla 3).

Mediante el método de Curvas ROC, se determinó el punto de corte para el EQ-SDAI en 13 pts (gráfico 1), obteniendo una alta sensibilidad $(\operatorname{Sen}=0,93)$, y una especificidad aceptable $(\mathrm{Esp}=0,60)$ (tabla 2).

A través del coeficiente de correlación biserial puntual, se obtuvo un resultado estadísticamente significativo $(\mathrm{R}>0,70)$, en ocho de las nueve preguntas de la escala SDAI, lo que indica que existe un buen coeficiente de determinación (tabla 4). La consistencia interna (KR20 ) fue de 0,866 ; considerada como buena.

Tabla 1. Comparación de la prevalencia de ansiedad, calculada mediante las escalas EQ-SDAI y SDAI.

\begin{tabular}{|c|c|c|c|c|c|c|c|c|c|c|c|c|}
\hline & \multicolumn{4}{|c|}{ EQ-SDAI } & \multicolumn{8}{|c|}{ SDAI } \\
\hline & \multicolumn{2}{|c|}{ Femenino } & \multicolumn{2}{|c|}{$\begin{array}{c}\text { Mascu- } \\
\text { lino }\end{array}$} & \multicolumn{2}{|c|}{ Total } & \multicolumn{2}{|c|}{ Femenino } & \multicolumn{2}{|c|}{ Masculino } & \multicolumn{2}{|c|}{ Total } \\
\hline & $\mathbf{n}$ & $\%$ & $\mathbf{n}$ & $\%$ & $\mathbf{n}$ & $\%$ & $\mathbf{n}$ & $\%$ & $\mathbf{n}$ & $\%$ & $\mathbf{n}$ & $\%$ \\
\hline Con Ansiedad & 309 & $83 \%$ & 244 & $75 \%$ & 553 & $79 \%$ & 316 & $85 \%$ & 256 & $79 \%$ & 572 & $82 \%$ \\
\hline Sin Ansiedad & 62 & $17 \%$ & 81 & $25 \%$ & 143 & $21 \%$ & 55 & $15 \%$ & 69 & $21 \%$ & 124 & $18 \%$ \\
\hline Total & 371 & $53 \%$ & 325 & $47 \%$ & 696 & $100 \%$ & 371 & $53 \%$ & 325 & $47 \%$ & 696 & $100 \%$ \\
\hline
\end{tabular}

Tabla 2. Tabla cruzada para determinar la sensibilidad y especificidad.

\begin{tabular}{|c|c|c|c|c|c|c|}
\hline \multicolumn{7}{|c|}{ DIAGNÓSTICO EQ- SDAI } \\
\hline \multirow[b]{2}{*}{ DIAGNÓSTICO SDAI } & \multicolumn{2}{|c|}{ Con Ansiedad } & \multicolumn{2}{|c|}{ Sin Ansiedad } & \multicolumn{2}{|c|}{ Total } \\
\hline & $\mathbf{n}$ & $\%$ & $\mathbf{n}$ & $\%$ & $\mathbf{n}$ & $\%$ \\
\hline Con Ansiedad & 512 & $93 \%$ & 60 & $42 \%$ & 572 & $82 \%$ \\
\hline Sin Ansiedad & 41 & $7 \%$ & 83 & $58 \%$ & 124 & $18 \%$ \\
\hline TOTAL & 553 & $79 \%$ & 143 & $21 \%$ & 696 & $100 \%$ \\
\hline
\end{tabular}

Tabla 3. Comprobación de diferencias de puntajes EQ-SDAI, entre los grupos de los niveles de la escala SDAI.

\begin{tabular}{|c|c|c|c|c|c|}
\hline \multicolumn{6}{|c|}{ HSD-Tukey B } \\
\hline & & 1 & 2 & 3 & 4 \\
\hline Sin Ansiedad & 124 & 0,468 & & & \\
\hline Levemente ansioso & 223 & & 2,000 & & \\
\hline Moderadamente ansioso & 198 & & & 4,177 & \\
\hline Extremandamente ansioso & 151 & & & & 7,450 \\
\hline
\end{tabular}




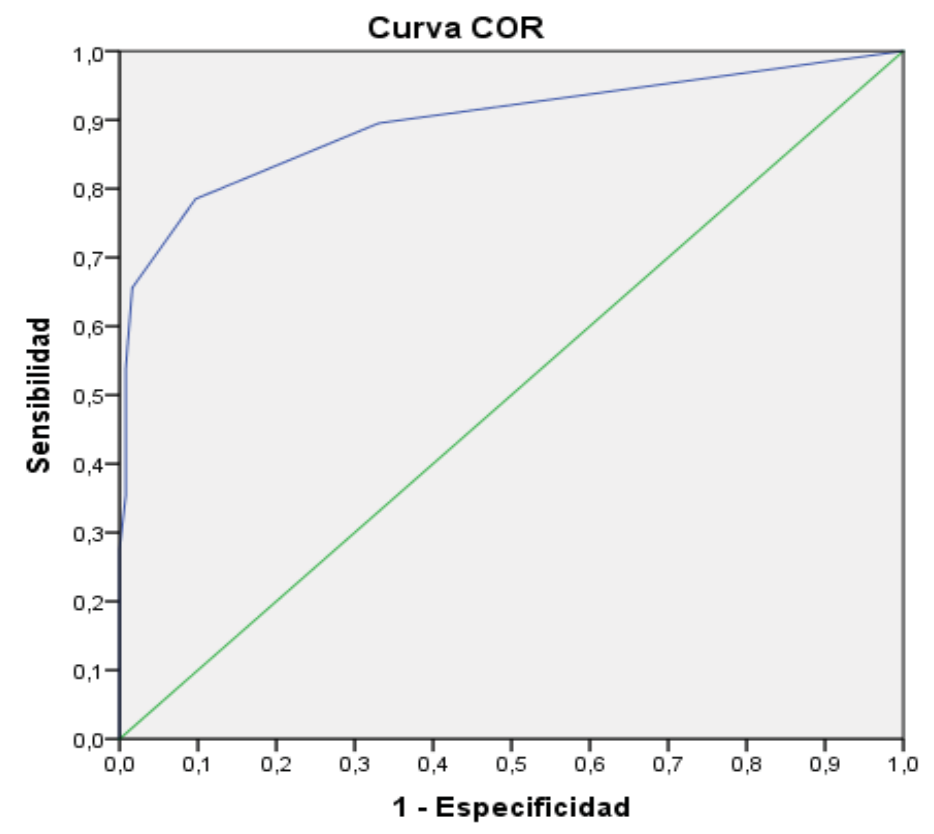

Los segmentos de diagonal se generan mediante empates.

Gráfico 1. Determinación del punto de corte apropiado según el método de Curvas ROC.

Tabla 4. Correlación biserial puntual.

\begin{tabular}{lcc}
\hline \multicolumn{1}{c}{ Preguntas de la escala SDAI } & $\mathbf{R}^{2}$ Pearson & $\begin{array}{c}\text { Coeficiente de } \\
\text { Determinación }\end{array}$ \\
\hline $\begin{array}{l}\text { 1. Comienzo a ponerme nervioso/a cuando el odontólogo me invita a sentarme en } \\
\text { la silla. }\end{array}$ & 0,69 & 0,83 \\
$\begin{array}{l}\text { 2. Cuando yo sé que el odontólogo va a extraerme un diente, me siento realmente } \\
\text { asustado en la sala de espera. }\end{array}$ & 0,57 & 0,75 \\
$\begin{array}{l}\text { 3. Cuando voy en camino al consultorio del odontólogo y pienso en el sonido de } \\
\text { la fresa, me dan ganas de devolverme y no ir. }\end{array}$ & 0,45 & 0,67 \\
$\begin{array}{l}\text { 4. Quiero irme del consultorio cuando pienso que el odontólogo no me va a expli- } \\
\text { car lo que hará en mis dientes. }\end{array}$ & 0,56 \\
$\begin{array}{l}\text { 5. En el momento en que el odontólogo alista la jeringa con la inyección de anes- } \\
\text { tesia, yo cierro mis ojos fuertemente. }\end{array}$ & 0,68 \\
$\begin{array}{l}\text { 6. En la sala de espera sudo y tiemblo cuando pienso que es mi turno de pasar a } \\
\text { la consulta. }\end{array}$ & 0,71 \\
$\begin{array}{l}\text { 7. Cuando voy hacia el consultorio del odontólogo, me pongo ansiosa sólo de } \\
\text { pensar si tendrá que usar la fresa conmigo. }\end{array}$ & 0,70 \\
$\begin{array}{l}\text { 8. Cuando estoy sentada en silla de tratamiento y no sé lo que el odontólogo está } \\
\text { haciendo en mi boca, me pongo nerviosa/o y sudo. }\end{array}$ & 0,84 \\
$\begin{array}{l}\text { 9. En mi camino hacia el consultorio del odontólogo, la idea de estar sentada en la } \\
\text { silla de tratamiento me pone nervioso. } \\
\text { TOTAL }\end{array}$ & 0,71 & 0,84 \\
\hline
\end{tabular}




\section{DISCUSIÓN}

El presente estudio se realizó en la ciudad de CuencaEcuador, con una muestra conformada por 371 mujeres y 325 hombres, la información se recolectó a través de encuestas, en pacientes con edades entre 18 y 65 años, donde se comparan los resultados obtenidos en la escala SDAI, frente al EQ-SDAI. Las prevalencias de Ansiedad Dental calculadas mediante la escala EQ-SDAI y SDAI no mostraron diferencia estadísticamente significativa $X^{2}(p>0,05)$. En la presente investigación tampoco se encontró diferencia estadísticamente significativa en la prevalencia de ansiedad dental calculada mediante ambas escalas en cada sexo.

Los resultados del cuestionario EQ-SDAI que tiene un formato dicotómico, no se alejan significativamente de los resultados de la escala SDAI politómica de 4 niveles de diagnóstico final (Sin ansiedad, levemente ansioso, moderadamente ansioso, extremadamente ansioso) (14,16), al momento de categorizar a los pacientes, esto se evaluó mediante la prueba ANOVA y luego se realizó el análisis post hoc HSD-Tukey.

La sensibilidad-especificidad del cuestionario EQ-SDAI se evaluó mediante el método de curva ROC. El punto de corte para esta validación fue de 13, tomado de los rangos estadísticos del SDAI descritos en el artículo de Caycedo et al., con el cual se comprobó a través del método de curvas ROC, una alta sensibilidad (Sen=0,93), y especificidad aceptable $(E s p=0,60)(16)$. Resultados semejantes se encontraron en el estudio de Fuentes y Cols., en el año 2014; que logran la validación del cuestionario de exposición a ruido alcanzando una sensibilidad del $78,57 \%$ y una especificidad del $62,86 \%$, considerada aceptable para su validación (13).

Con un punto de corte de 14 se lograrían 4 puntos menos en la sensibilidad, pero se ganan 11 puntos en la especificidad, sin embargo, no hay literatura que respalde este cambio en el punto de corte, por lo tanto, este cambio queda como propuesta para próximas investigaciones. De igual forma se analizó la posibilidad de eliminar la pregunta tres que alcanzó un coeficiente de determinación de 0,67 de correlación en la consistencia interna, sin embargo, este cambio alteraba la sensibilidad y especificidad total del instrumento, disminuyéndolas, por lo que se consideró necesario mantener las nueve preguntas del SDAI versión corta.

En el presente estudio se obtuvo un Coeficiente de Kuder Richardson $=0,86$, este coeficiente es una particularidad del procedimiento de Alfa de Cronbach para evaluar la consistencia interna de los instrumentos con variables dicotómicas. El valor de KR-20 hallado en el presente estudio se considera como un buen nivel de consistencia, dato que es concordante con otros estudios como el de la consistencia interna de validación de la Escala de Ansiedad Dental versión corta (MDAS) italiana, realizada por Gremigni et al., en el año 2014; que logró un Alfa de Cronbach de 0,87 considerado bueno (35). Otro estudio de validación con el que se obtienen resultados similares es el estudio de Bhayat et al., en el año 2014; que validó la versión corta en árabe del cuestionario de calidad de vida relacionado con la salud bucal infantil (CPQ 11-14), que obtuvo un Alfa de Cronbach de 0,82 ; investigación que también considera que su nivel de consistencia interna es bueno (36). En algunas investigaciones se reportan valores más elevados de Alfa de Cronbach que bordean el 0,90 y que al igual que la presente investigación todavía se consideran dentro del nivel bueno de consistencia interna, como es el caso de la investigación realizada por Azza et al., en el año 2016; que validó la escala para medir el miedo infantil a través de la encuesta: subescala dental para niños de habla árabe, con alto Alfa de Cronbach $>0,9$ (37). De igual manera el trabajo realizado por Castrejón R. y Cols., en el año 2012; para la validación de la forma corta del perfil de impacto de salud oral en español (OHIP-EE-14), obtuvo un Alfa de Cronbach de 0,91 considerado bueno (38). Es importante destacar que existen estudios con un Alfa de Cronbach $\geq 0,7$, considerado aceptable para la validar instrumentos, como es el caso de los estudios realizados por Lea Kragt et al., en el año 2015 (39), y Zucoloto et al., en el año 2014 (40); lo cual nos refiere que hay instrumentos validados inclusive con valores inferiores a los obtenidos en el presente estudio.

Dado que el foco de atención del presente estudio es el cálculo de la prevalencia, y que este análisis se realizó solamente en población ecuatoriana, se considera que una limitación del presente estudio es su aplicabilidad a otros países de habla hispana, por lo cual se recomienda, replicar este estudio en diferentes poblaciones. En conclusión, la versión 
EQ-SDAI, basada en la escala de ansiedad dental SDAI validada por Artmant (14), tiene características psicométricas que permiten su aplicación para el cálculo de la prevalencia de la ansiedad dental en estudios epidemiológicos de campo. Se propone esta nueva forma de evaluar la Ansiedad Dental, como herramienta para levantar datos de línea base que permitan plantear programas de intervención en la comunidad.

\section{AGRADECIMIENTOS}

Los autores agradecen a la Dra. Irene Aartman, por el permiso para hacer la validación y adaptación transcultural de su instrumento, el cual fue dicotomizado para ser utilizado en estudios comunitarios en Latinoamérica.

\section{Correspondencia:}

Ebingen Villavicencio Caparó

Correo electrónico: evillavicencioc@ucacue.edu.ec

\section{REFERENCIAS}

1. World Health Organization. Oral health surveys: basic methods. Ginebra: World Health Organization; 2013. (Citado el 12 de junio del 2018) Disponible en: http:// www.who.int/oral_health/publications/ 9789241548649/en/

2. Villavicencio E, Ruiz V, Cabrera A. Validación de cuestionarios. Odontología Activa. 2016; 1(3):0-0.

3. Aday L, Cornelius L. Designing and conducting health surveys: A comprehensive guide. San Francisco: Jossey-Bass; 2006.

4. Mokkink L, Terwee C, Patrick D, et al. The Cosmin study reached international consensus on taxonomy, terminology, and definitions of measurement properties for health-related patient-reported outcomes. J Clin Epidemiol. 2010; 63(7):737-45. doi: 10.1016/j. jclinepi.2010.02.006

5. Argimon J, Jiménez J. Métodos de investigación clínica y epidemiológica. Madrid: Elsevier; 2004.

6. Müller R, Büttner P. A critical discussion of intraclass correlation coefficients. Stat Med. 1994;13(2324):2465-76.

7. Choi B, Granero R, Pak A. Catálogo de sesgos o errores en cuestionarios sobre salud. Revista Costarricense de Salud Pública. 2010; 19(2): 106-118

8. Cuauhtémoc V. Estadística descriptiva y selección de la prueba. Rev Mex Cardiol. 2014;25(2): 129-131.

9. Cumsille F, Bangdiwala S. Categorización de variables en el análisis estadístico de datos: consecuencias sobre la interpretación de resultados. 2000; 8(5):348-354.

10. Ragland D. Dichotomizing continuous outcome variables: dependence of the magnitude of association and statistical power on the cutpoint. Epidemiology. 1992;3(5):434-40.

11. López J. Îtems politómicos vs. dicotómicos: Un estudio metodológico. Anales de Psicología. 2005;21(2): 339344.

12. Burriel J, Chesa M, Tena V. Construcción y validación psicométrica de un cuestionario de actitudes hacia el uso del valenciano en la universidad. Castelló de la Plana: Universitat Jaume I; 1995. (Citado el 12 de junio del 2018) Disponible en: http://repositori.uji. es/xmlui/bitstream/handle/10234/80568/ forum_1995_8.pdf? sequence $=1$

13. Fuentes E, Cardemil F. Validación de criterio y constructo para la creación de un cuestionario de exposición a ruido. Revista de otorrinolaringología y cirugía de cabeza y cuello. 2014; 74: 21-30

14. Aartman I. Reliability and validity of the short version of the Dental Anxiety Inventory. Community Dent Oral Epidemiol. 1998;26(5):350-4.

15. Lima M, Casanova Y. Miedo, ansiedad y fobia al tratamiento estomatológico. Revista de Humanidades Médicas. 2006; 6(1):0-0.

16. Caycedo C, Cortés O, Gama R, et al. Ansiedad al tratamiento odontológico: Características y diferencias de género. Suma Psicológica. 2008; 15(1): 259-278.

17. Villavicencio-Caparó E. Barreras para el acceso a servicios de salud bucal. Odontología Activa. 2017; 2(2):1-6.

18. Márquez J, Navarro M, Cruz D, Gil J. ¿Por qué se le tiene miedo al dentista? RCOE. 2004; 9(2): 165-174

19. Valenzuela L, Cifuentes L. Validez de estudios de tests diagnósticos. Rev med Chile. 2008; 136(3): 401-404.

20. Restrepo L, González J. De Pearson a Spearman. Rev Colom Cienc Pecua. 2007; 20(2): 183-192.

21. Saunders D, Trapp R. Bioestadística Médica. Ciudad de México: El Manual Moderno; 1996.

22. Martín A, Luna J. Bioestadística para las ciencias de la salud. Madrid: Editorial Norma; 1993.

23. Segura J. Sensibilidad y especificidad de los métodos. RCOE. 2002; 7(5):491-501.

24. Flores A, Gómez F, Valles R, Rodríguez G, Flores S, Alonso R. Sensibilidad y especificidad del diagnóstico clínico de cardiopatías congénitas. Revista Mexicana de Pediatría. 2002; 69(3): 99-101.

25. Castro L, Rodríguez J, Ronquillo M, et al. Sensibilidad y especificidad de la prueba cutánea por punción con extractos alergénicos estandarizados de Dermatophagoides pteronyssinus en adultos. Vaccimonitor. 2013; 22(2): 24-29.

26. McHugh ML. The Chi-square test of independence. Biochemia Medica. 2013; 23(2):143-149. 
27. Cerda J, Cifuentes L. Uso de curvas ROC en investigación clínica: Aspectos teórico-prácticos. Rev chil infectol. 2012;29(2):138-141.

28. Akobeng A. Understanding diagnostic tests 3: Receiver operating characteristic curves. Acta Paediatr. 2007;96(5):644-7.

29. Altman D, Bland J. Diagnostic tests 3: receiver operating characteristic plots. BMJ. 1994 Jul;309(6948):188.

30. Robertson E, Zweig M. Use of receiver operating characteristic curves to evaluate the clinical. Clin Chem. 1981; 27(9):1569-74.

31. Martínez P. Comparación de pruebas diagnósticas desde la curva ROC. Rev Colomb Estad. 2007; 30(2): 163-176.

32. Celina H, Campo A. Aproximación al uso del coeficiente Alfa de Cronbach. Rev Colomb Psiquiatr. 2005; 34(4): 572-580.

33. da Silva F, Gonçalves E, Arancibia B, et al. Estimadores de consistencia interna en las investigaciones en salud: En el uso del coeficiente Alfa. Rev perú med exp salud pública. 2015; 32(1): 129-138.

34. Cappello G, Aguirre M, Castro T, Cervantes C, Marín A. Estudio del análisis de la consistencia interna y la validez de construcción de una prueba en el programa de enseñanza vivencial de las ciencias. Revista Internacional de Ciencias Sociales y Humanidades, SOCIOTAM. 2004; 14(2):47-72.
35. Gremigni P, Mobilio N, Casu G, Catapano S. Validation of the Modified Dental Anxiety Scale (MDAS) in an Italian Sample and Invariance Across Gender and Mode of Administration. European Journal of Psychological Assessment. 2014;30(2):140-49.

36. Bhayat A, Ali M. Validity and reliability of the Arabic short version of the child oral health-related quality of life questionnaire (CPQ 11-14) in Medina, Saudi Arabia. Eastern Mediterranean Health Journal. 2014; 20(8):477-82.

37. Azza A, Farah A, Najlaa M, et al. Reliability and validity of the Children's Fear Survey Schedule-Dental Subscale for Arabic speaking children: a cross-sectional study. BMC Oral Health. 2016; 16(49):0-0.

38. Castrejón R, Borges A. Derivation of the short form of the Oral Health Impact Profile in Spanish (OHIPEE-14). Gerodontology. 2012;29(2):155-8.

39. Kragt L, Tiemeier H, Wolvius E, Ongkosuwito E. Measuring oral health-related quality of life in orthodontic patients with a short version of the Child Oral Health Impact Profile (COHIP). Journal of Public Health Dentinstry. 2015; 76(2): 105-112.

40. Zucoloto M, Maroco J, Campos J. Psychometric properties of the oral health impact profile and new methodological approach. J Dent Res. 2014;93(7):645-50.

Recibido: 24-04-2019

Aceptado: 16-08-2019 